\title{
Mobile technology intervention to improve care coordination between HIV and substance use treatment providers: development, training, and evaluation protocol
}

\author{
Kasey Claborn ${ }^{1,2^{*}}$, Sara Becker ${ }^{2,3}$, Susan Ramsey ${ }^{1,2}$, Josiah Rich ${ }^{2,4}$ and Peter D. Friedmann ${ }^{5}$
}

\begin{abstract}
Background: People living with HIV (PLWH) with a substance use disorder (SUD) tend to receive inadequate medical care in part because of a siloed healthcare system in which HIV and substance use services are delivered separately. Ideal treatment requires an interdisciplinary, team-based coordinated care approach, but many structural and systemic barriers impede the integration of HIV and SUD services. The current protocol describes the development and preliminary evaluation of a care coordination intervention (CCI), consisting of a tablet-based mobile platform for HIV and SUD treatment providers, an interagency communication protocol, and a training protocol. We hypothesize that HIV and SUD treatment providers will find the CCI to be acceptable, and that after receipt of the CCI, providers will: exhibit higher retention in dual care among patients, report increased frequency and quality of communication, and report increased rates of relational coordination.
\end{abstract}

Methods/design: A three phase approach is used to refine and evaluate the CCI. Phase 1 consists of in-depth qualitative interviews with 8 key stakeholders as well as clinical audits of participating HIV and SUD treatment agencies. Phase 2 contains functionality testing of the mobile platform with frontline HIV and SUD treatment providers, followed by refinement of the CCI. Phase 3 consists of a pre-, post-test trial with 30 SUD and 30 HIV treatment providers. Data will be collected at the provider, organization, and patient levels. Providers will complete assessments at baseline, immediately post-training, and at 1-, 3-, and 6-months post-training. Organizational data will be collected at baseline, 1-, 3-, and 6-months post training, while patient data will be collected at baseline and 6-months post training.

Discussion: This study will develop and evaluate a CCl consisting of a tablet-based mobile platform for treatment providers, an interagency communication protocol, and a training protocol as a means of improving the integration of care for PLWH who have a SUD. Results have the potential to advance the field by bridging gaps in a fragmented healthcare system, and improving treatment efficiency, work flow, and communication among interdisciplinary providers from different treatment settings.

Trial Registration: NCT02906215

Keywords: HIV, Addiction, Communication, Mobile technology, Treatment providers

\section{Background}

Alcohol and illicit drug use remains a substantial problem among people living with HIV (PLWH), contributing to

\footnotetext{
*Correspondence: kasey_claborn@brown.edu

${ }^{1}$ Department of Medicine, Division of General Internal Medicine, Rhode Island Hospital, 111 Plain Street, Providence, RI 02903, USA

Full list of author information is available at the end of the article
}

suboptimal treatment outcomes and increased transmission of HIV. Over $81 \%$ of PLWH report use of an illicit drug, and nearly one in four meet diagnostic criteria for a substance use disorder (SUD) [1]. Substance use is associated with poorer outcomes across the HIV care continuum including delayed diagnosis and linkage to HIV care, decreased adherence to antiretroviral medications,

\section{(


reduced retention in care, increased sexual risk behavior leading to inferior clinical outcomes, and increased HIV transmission and drug resistance [2]. HIV-infected people who use drugs are more likely to have medical (e.g., hepatitis C, tuberculosis) and psychiatric (e.g., depression, anxiety) comorbidity, neurocognitive impairment [3], and increased risk for drug overdose [4], resulting in increased utilization of services, morbidity, and mortality [5-7]. Further, this population tends to cycle in and out of treatment, resulting in suboptimal health outcomes $[8,9]$. PLWH who have a SUD tend to receive inadequate medical care [10] which is in part a byproduct of traditional HIV care and substance use services being delivered separately [11].

The Institute of Medicine has recommended integrated care between substance use disorder (SUD) treatment and primary care since 2006 [12, 13]; however, there remains a dearth of evidence in regards to the optimal method of service integration. Both HIV-infection and SUDs are chronic and treatable diseases requiring continued care [13]. With higher rates of SUDs among HIVinfected populations [2], early identification of SUDs and referral to treatment is vital to effective clinical management and prevention of HIV transmission. An interdisciplinary, comprehensive, team-based coordinated care approach has been identified as the ideal treatment for PLWH who have a SUD [13]. Benefits of service integration have been noted at the patient-, provider-, and societal-levels, including decreased healthcare costs and improved treatment outcomes [14-16]. Ideal treatment includes an interdisciplinary team-based approach which can improve tracking and monitoring of patients, and coordination of comprehensive treatment plans, which may prevent treatment drop-out or expedite patient reengagement in care [16].

Many structural and systemic barriers impede the integration of treatment for HIV and SUDs. Start-up costs and limited building space are frequent deterrents of such integration. Consequently, patients with comorbid diagnoses tend to be referred to multiple providers at offsite clinics and are often lost in the referral process. Further, effective integration and quality treatment require additional staff and provider training in both HIV disease and substance use treatment [17]. Montague et al. [18] identified several gaps in care among 119 HIV treatment providers and 159 SUD treatment providers. Results demonstrated that providers need cross-training to increase knowledge of risks associated with these comorbid conditions and require training in effective assessment and identification for both diseases. Additional training needs included education about available referral sources for dual treatment and the need to define the role of SUD treatment providers in supporting HIV care
[18]. Co-management of HIV and SUDs hinges on effective communication between a team of interdisciplinary providers. Current infrastructure frequently limits timely information transfer, resulting in deficits in communication. Further, cultural and language differences within the independent organizational structures and providerrelated stigma are important barriers to integrating HIV and SUD treatment [19]. There exists an urgent need for the healthcare infrastructure to adequately address the needs of dually diagnosed patients in a cost-effective and easily implemented manner. Information technology solutions designed for providers may address many of these barriers to dual care.

Rudin and Bates [20] identified four areas within a care coordination framework that would likely benefit from information technology solutions: (1) the ability to identify a patient's care team both within and across settings and disciplines (including primary care providers, specialists, social worker, case manager, substance use counselor), (2) the ability to collaborate with the care team in a quick and efficient manner, (3) the ability to collaborate through the sharing and formulation of care plans, and (4) the ability to monitor and track task responsibilities [20]. However, electronic health record (EHR) systems still lag in several areas including: information exchange across settings; development, storing, and sharing of care plans; tracking of referrals; and improved team-based communication [21].

A preliminary study conducted by the investigative team highlighted HIV and addiction treatment provider perspectives in regards to care coordination barriers and strategies to improve coordination [22]. In-depth qualitative interviews revealed concerns that EHRs, as they are currently designed, may result in poorer comprehensive care for PLWH who have a SUD as a result of stigma related to the documentation of both HIV disease and substance use. Providers expressed a desire for development of an integrated, secure technology platform specifically designed for HIV and addiction providers as a solution to improved communication and care coordination across disciplines. Recommended features included an instant messaging system, identification of the patient's care team and contact information, real-time notifications, HIV and SUD training resources, and community resources. Providers conveyed interest in a range of potential delivery modalities including a web-based platform and mobile platforms accessed through tablet or mobile phone devices. When asked explicitly what platform they would prefer, a majority of providers preferred a tablet- or phone-based mobile platform, due to the perceived benefits of increased mobility provided by these devices. Additional perceived benefits included the capability to interact with patients with the technology 
interface on a tablet device during appointments and potential for features to allow real-time notifications and easy access to data (e.g., have alarms for notification if a high-risk patient missed appointments or is admitted into the emergency department). Some providers noted, however, that they would most value a mobile platform if it could either be co-located (i.e., stored on the same workstation) or fully integrated (i.e., embed within) the EHR in order to minimize duplication of data entry, highlighting the need for a tool that can be flexibly delivered to meet provider preferences.

Mobile health technology offers a unique potential platform for rapid communication among providers and tracking of patient service utilization and treatment approaches. Additionally, it has the potential to improve the team-based approach consistent with the collaborative care model, while promoting more effective care in hard-to-retain populations that place a significant burden on the healthcare system, such as PLWH who have a SUD. Currently, there are over 200 HIV-related mobile health applications marketed for PLWH on either android or apple platforms. A 2013 review of mobile applications found that only 55 apps promoted HIV prevention and care services, and the majority of these apps focused on providing disease-specific educational information only [23]. No mobile applications were identified for treatment providers. Mobile applications are unique among devices and tools used in clinical practice in that there is currently no mechanism for regulating them or ensuring they are efficacious or beneficial. A theorydriven and empirically tested technology platform for providers that over the long-term be flexibly delivered as either a stand-alone product or integrated with the EHR has the potential to improve coordination and treatment management of dually diagnosed patients.

For the purposes of this study, care coordination is defined as "the deliberate organization of patient care activities between two or more treatment settings [HIV clinic, SUD clinic] involved in a patient's care to facilitate the appropriate delivery of health care services; organizing care involves the marshalling of personnel and other resources needed to carry out all required patient care activities and is often managed by the exchange of information among participants responsible for different aspects of care" (p. 2) [24]. Van Houdt et al. [24, 25] developed a theoretical framework of care coordination which encompasses organizational-, provider-, and patient-level characteristics (see Fig. 1). This framework will guide the development of a technology-assisted care coordination intervention (CCI) for HIV and substance use treatment providers. Consistent with the Care Coordination framework, the CCI seeks to improve relational coordination components through modification of the inter-organizational mechanisms of structure, knowledge and information technology, administrative operational processes, and cultural factors. A wealth of literature has

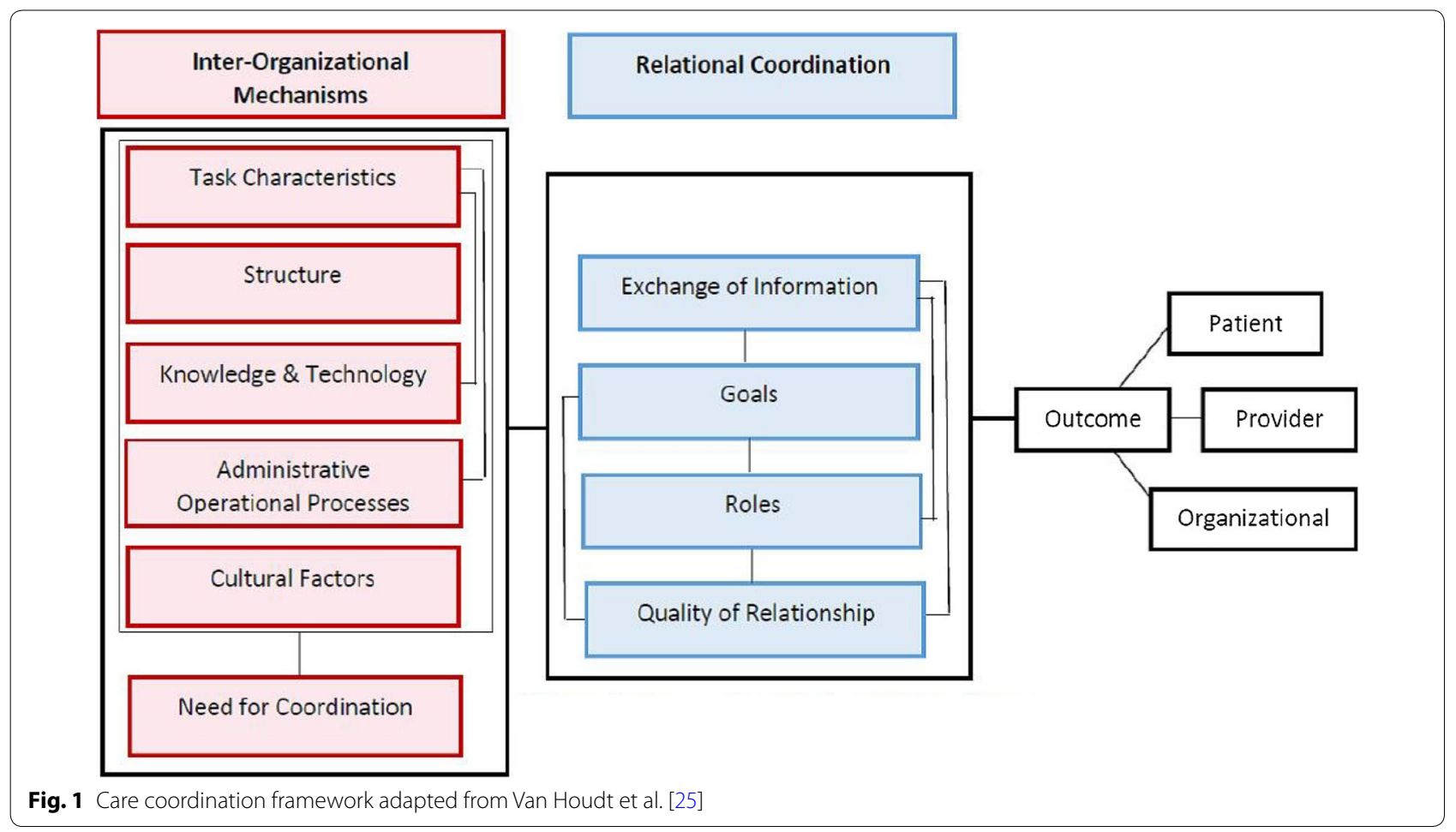


demonstrated that didactic training-alone is insufficient to produce optimal change $[26,27]$. Thus, we will use an evidence-based comprehensive training approach to promote adoption of the CCI that addresses both organizational and individual provider level factors [28-30].

\section{Present study}

This study uses mixed methods to optimize care coordination of patients diagnosed with HIV and substance use issues using an organizational-level intervention that combines an evidence-based training approach with use of mobile technology. The proposed project consists of three phases. In Phase 1, we will use qualitative data to guide the development of the CCI and training approach. Phase 2 will consist of a series of functionality tests of the tablet-based mobile application prototype using fake patient data with HIV and SUD treatment providers, and a review of the intervention with a stakeholder panel of clinic leadership. Data regarding feasibility, acceptability, and barriers that would limit effectiveness of the intervention will guide modifications to finalize the software and the training protocol. In Phase 3, we will conduct a pre-, post-test trial of the CCI to examine its effects on the amount and quality of interagency communication, perception of interagency professional relationships, and retention of patients in dual care. Preliminary data regarding intervention costs and sustainability potential will be collected. Table 1 depicts the timing of key protocol elements for the Phase 3 pilot trial including provider enrollment, provider training, and assessments.

The primary goal of this protocol is to develop and test a CCI among HIV and SUD treatment providers. We hypothesize that the combination of training and mobile health tools specifically created for HIV and SUD care coordination will (a) increase patient retention in dual care, (b) increase the amount of communication between
HIV and addiction treatment providers, and (c) improve relational coordination between HIV and SUD treatment providers.

\section{Methods \\ Participants and setting}

Two organizations in the Northeast region of the United States, an academic affiliated HIV clinic and a non-profit SUD treatment facility, will serve as the recruitment and implementation sites for the CCI. The HIV clinic is funded by the Ryan White program and provides comprehensive care for over $1600 \mathrm{HIV}$-infected patients. The clinic also has a pre-exposure prophylaxis (PrEP) program which offers a single, daily pill to patients at higher risk for HIV exposure to prevent the person from being infected with HIV. The collaborating SUD treatment program is a non-profit organization that provides substance use treatment, recovery, and prevention services to about 950 new patients annually across four clinics. These services include general outpatient (drug free) or intensive outpatient counseling for alcohol and drug-related problems.

Key stakeholders ( $\mathrm{n}=8$ in Phase 1: stakeholder interviews) and frontline HIV and SUD treatment providers ( $\mathrm{n}=8$ in Phase 2: functionality testing; and $\mathrm{n}=60$ in Phase 3: Pilot Trial) will be recruited from the HIV and SUD clinics. Upper management in each organization will inform providers that participation in the study is available and they may volunteer to participate; providers will be explicitly informed that participation is voluntary and will not affect their employment. Once a potentially eligible provider has been identified, the RA will contact the provider, introduce the study, and assess eligibility. For inclusion, key stakeholders must: (a) be at least 18 years of age, (b) have a major administrative or leadership role (i.e., President/CEO, Clinic Director, Executive

Table 1 Timing of provider enrollment, training, and assessment activities

\begin{tabular}{|c|c|c|c|c|c|c|}
\hline \multirow[t]{2}{*}{ Timepoint } & \multirow{2}{*}{$\begin{array}{l}\text { Enrollment } \\
-\mathrm{t} 1\end{array}$} & \multirow{2}{*}{$\begin{array}{l}\text { Training } \\
0\end{array}$} & \multicolumn{4}{|c|}{ Post-didactic training } \\
\hline & & & Post training & +1 month & +3 months & +6 months \\
\hline \multicolumn{7}{|l|}{ Provider enrollment } \\
\hline Informed consent/assent & $x$ & & & & & \\
\hline \multicolumn{7}{|l|}{ Training } \\
\hline Didactic sessions \#1-3 & & $x$ & & & & \\
\hline Interagency communication manual & & $x$ & & & & \\
\hline Internal change champions & & & $x$ & $x$ & $x$ & $x$ \\
\hline External leadership coaching & & & $x$ & $x$ & $x$ & $x$ \\
\hline \multicolumn{7}{|l|}{ Assessments } \\
\hline Provider-level outcomes & $x$ & & $x$ & $x$ & $x$ & $x$ \\
\hline Organizational-level outcomes & $x$ & & & $x$ & $x$ & $x$ \\
\hline Patient-level outcomes & $x$ & & & & & $x$ \\
\hline
\end{tabular}


Leadership) in an HIV or SUD treatment center, (c) have been employed in their current leadership position for at least 6 months, and (d) able and willing to provide informed consent. Treatment providers must: (a) be at least 18 years of age, (b) provide either HIV care or SUD treatment [e.g., physicians, residents, psychologists, midlevel providers], (c) have been employed at a participating recruitment site for at least 6 months, (d) have no plans for a leave of absence over the next 2 years and (e) willing and able to provide informed consent. We will recruit an even number of stakeholders and providers from HIV and SUD treatment centers. We recognize that staff turnover may occur throughout the study and especially during Phase 3 (6-month intervention phase). Providers who leave employment during the trial period will be asked to return the tablet device to the study and will be removed from participating in the $\mathrm{CCI}$ for the remainder of the study. Participants in Phase 1 (stakeholder interviews) will receive $\$ 75$, and participants in Phase 2 (functionality testing) will be paid $\$ 50$. In Phase 3 (pilot trial), participants will be paid $\$ 50$ for completion of the baseline interview and $\$ 40, \$ 45$, and $\$ 50$, respectively, for completion of the 1-, 3- and 6-month follow-ups. Participants completing exit interviews will receive an additional $\$ 50$.

\section{Procedures \\ Phase 1: qualitative methods to inform intervention development}

Individual interviews with 8 key stakeholders and clinic audits will be used to inform development of the CCI. All qualitative interviews will follow a semi-structured agenda covering the following topics: (a) interagency processes that facilitate or impede interagency collaboration; (b) organizational resources available to support interagency collaboration; (c) professional knowledge domains and boundaries (i.e., substance use providers knowledge of HIV care and vice versa); (d) expectations in regards to authority to coordinate treatment; (e) issues of patient confidentiality in regards to coordination of HIV and SUD care. Interviews will last approximately $60 \mathrm{~min}$, and participants will receive $\$ 75$. We will regularly assess data saturation on key topics and may conduct a higher number of interviews if more information is required. In addition, the CCI will be informed by 29 individual interviews with HIV and SUD treatment providers that were conducted as part of another NIDA funded study (K23DA039037). The goal of this phase is to gather organizational-level data to evaluate potential barriers and facilitators to collaboration within each clinic setting.

Audit of referral and communication process To augment the stakeholder interviews, audits within each clinic will be conducted to assess existing referral and commu- nication procedures. Data to be extracted include number of relevant referrals by provider type, number of patients followed post-referral, and language used in referrals and consult letters. These data will inform the interagency communication protocol and language for the CCI.

Coding and analysis Qualitative data will be analyzed using a thematic framework method [31]. This method will allow the investigative team to develop themes from both the research questions and the narratives of the research participants [32]. A preliminary coding structure will be derived deductively from the interview script, with specific subtype coding applied inductively as themes and repetitions emerge from the data. Data analysis will be iterative using standard analysis techniques, including open coding, axial coding, marginal remarks, and memowriting [33]. Preliminary research questions include: (1) what are the gaps in existing referral and communication processes; (2) how do providers exchange patient information; (3) how do providers perceive the need for care coordination; and (4) what is the level of cross-training and available resources.

\section{Phase 2: intervention and manual development \& refinement} Based on data triangulated from Phase 1 and evaluation of the literature, we will develop the CCI prototype and refine the training and intervention protocols. The development of the CCI will involve a series of meetings among the investigators and the software development team to review the core elements and features of the mobile application. The goal of these meetings will be to match the intervention content and structure to the needs and preferences identified through formative research.

Intervention The CCI will consist of a mobile application developed for treatment providers, an interagency communication protocol, and a training protocol. The mobile application will be developed on a secure portable platform that providers will access through study-provided tablet devices. The CCI dashboard will be user friendly and easily navigable. The application will feature a secure messaging center for providers to directly communicate. Other application features will include: (a) resources for methods of identifying patients in need of dual care, (b) a system to refer patients to treatment, (c) ability to track patients' access and utilization of services, (d) real-time patient care notifications, (e) identification of a patient's care team, and (f) upto-date lists of resources and evidence-based recommendations for treatment options. The CCI will provide a secure platform that will enable the transfer of patient health information between providers, yet will not be linked in the same electronic health record system, adding an additional level 
of privacy for patients who do not wish for non-HIV/SUD providers to have access to this sensitive data. These features of the CCI prototype are based on preliminary work [22]; however, the final app will reflect data gathered from the Phases 1 and 2 of the study.

Refinement of the mobile application and training materials Once the prototype $\mathrm{CCI}$ and training materials have been developed, these materials will be refined through an iterative process whereby we will test the functionality of the CCI with 6-8 providers. During these individual interviews, we will demonstrate the CCI features and solicit feedback on the training procedures and the interagency communication protocol. These data will inform modifications to the CCI. Once modifications have been completed, the investigative team will have a 2 -h (in-person/teleconference/Skype) meeting with the 8 stakeholders who participated in Phase 1 to review modifications and finalize the CCI.

\section{Phase 3: provider training and intervention testing}

Phase 3 will test the organizational intervention. We recognize use of the mobile application alone is likely to yield minimal improvements in care coordination. Consequently, the proposed intervention augments the mobile application with training of providers and ongoing support for training needs.

Training procedures Although the primary aim of the proposed study is intervention development, we are mindful of the need to have an evidence-based replicable training protocol to facilitate widespread implementation. We use the Service to Science Laboratory (SSL) staff training model, which was specifically developed by the SAMHSA-funded Addiction Technology Transfer Centers (ATTCs) to increase support for intervention adoption at both the individual-level and organizational-level. The SSL model contains three key elements: upfront didactic training, ongoing performance feedback, and ongoing coaching from an external leadership coach who works with Internal Change Champions within each organization [34].

The upfront didactic training will be led by a member of the New England ATTC staff and will consist of three training sessions (see Table 2 for an overview of topics). Participants will complete a 20-item baseline knowledge assessment prior to Training Session \#1. All training sessions will be audio-recorded and participants who are unable to attend will be required to listen to the audio recording and attend a brief make-up training session. Upon completion of Training Session \#3, each treatment provider will be required to complete a 20 -item posttraining knowledge assessment and to demonstrate adequate knowledge, defined as accurate answers to $16(80 \%)$ of the items. Each provider will also complete a role play with the ATTC trainer using a standardized patient case. During the role play, the provider will be required to demonstrate competence in the following six areas: (a) identifying resources to detect patients in need of dual care, (b) using the referral system (including ability to

Table 2 Expected features and benefits of the care coordination intervention (CCI) based on preliminary qualitative work

\begin{tabular}{|c|c|c|c|c|}
\hline \multicolumn{5}{|l|}{ Training procedures } \\
\hline \multicolumn{2}{|c|}{ Interagency communication protocol } & \multicolumn{3}{|c|}{$\begin{array}{l}\text { Include a manual for the mobile app and outline communication procedures and } \\
\text { language }\end{array}$} \\
\hline \multicolumn{2}{|c|}{ Didactic Session 1 (conduct separately at each clinic) } & \multicolumn{3}{|c|}{$\begin{array}{l}\text { Provide study details, solicit buy-in and commitment to change, cross-training in HIV/ } \\
\text { SU issues }\end{array}$} \\
\hline \multicolumn{2}{|c|}{ Didactic Session 2 (conduct concurrently at neutral location) } & \multicolumn{3}{|c|}{ Train in CCl and study procedures, introduce participants from each clinic } \\
\hline \multicolumn{2}{|c|}{ Didactic Session 3 (conduct separately at each clinic) } & \multicolumn{3}{|c|}{$\begin{array}{l}\text { Hands-on practice with the mobile application using fake patient data, pass a CCI } \\
\text { competency test }\end{array}$} \\
\hline \multicolumn{2}{|c|}{ Science to Service Laboratory (ATTC) } & \multicolumn{3}{|c|}{$\begin{array}{l}\text { Appoint Internal Implementation Champions within each clinic; external leadership } \\
\text { coach }\end{array}$} \\
\hline \multicolumn{5}{|l|}{ Mobile application features } \\
\hline $\begin{array}{l}\text { Enable rapid communica- } \\
\text { tion }\end{array}$ & Patient linkage to dual care & Care coordination & Work flow & Dual care resources \\
\hline Portable platform & $\begin{array}{l}\text { Identification of patients in } \\
\text { need }\end{array}$ & Identification of care team & $\begin{array}{l}\text { Direct referrals from provid- } \\
\text { ers }\end{array}$ & Patient educational resources \\
\hline Secure messaging center & Easy referral system & $\begin{array}{l}\text { Easily develop dual care } \\
\text { plans }\end{array}$ & $\begin{array}{l}\text { Manage transitions to dual } \\
\text { care }\end{array}$ & $\begin{array}{l}\text { Provider educational } \\
\text { resources }\end{array}$ \\
\hline $\begin{array}{l}\text { Notification of message } \\
\text { receipt }\end{array}$ & $\begin{array}{l}\text { Track patient's service } \\
\text { usage/appointment } \\
\text { attendance }\end{array}$ & $\begin{array}{l}\text { Communicate with care } \\
\text { team via SMS procedures }\end{array}$ & $\begin{array}{l}\text { Decrease time spent on } \\
\text { coordinating care }\end{array}$ & $\begin{array}{l}\text { Available community } \\
\text { resources and admission } \\
\text { criteria }\end{array}$ \\
\hline Remote access to system & Link to HIV/PrEP/SU services & Track and manage dual care & Decision trees for referrals & Identify provider availability \\
\hline
\end{tabular}


both make a referral and accept a referral request), (c) tracking of patients' access and utilization of services, (d) reading real-time patient care notifications, (e) identifying a patient's care team, and (f) reviewing up-to-date lists of resources and evidence-based recommendations for treatment options. Providers who do not demonstrate competence will receive immediate corrective feedback and up to two additional role plays will be conducted until competence is shown. Only those providers who exhibit competence in all six areas will proceed to the next phase of training.

After completion of the 3-session didactic training series, we will enable the mobile application, assign each provider a tablet device, and initiate the 6-month open trial. At this point, the SSL training model will shift from a focus on didactics to ongoing interpersonal support. The SSL relies heavily on supportive interpersonal personal strategies through the use of an external leadership coach and Internal Change Champions, who continually encourage collaborative problem solving throughout the organization. The eight key stakeholders will be responsible for either serving as Internal Change Champions or selecting alternates. The external leadership coach will have monthly coaching calls with the Internal Change Champions focused on tracking implementation progress and engaging in problem solving to address barriers to sustained use of the mobile application. In turn, the Internal Change Champions will be responsible for providing ongoing supervision and support to frontline treatment providers. Consistent with the recommendations of Montague et al. [18], who noted the need for cross-training among HIV and substance use providers, the SSL will include cross-training via explicit didactic instruction and ongoing coaching in an effort to increase understanding of each provider's role and disease specific processes.

Measures The framework identified in Fig. 1 has informed the battery of measures. Assessments will focus on organizational-, provider-, and patient-level domains (see Table 3). Primary outcomes will be feasibility and acceptability of the CCI and assessment procedures to be used in a larger trial [35]. Organizational outcomes will include pre- and post-intervention assessments of each clinic to gather data on organizational structure and procedures for care coordination. Provider outcomes will include feedback on the intervention component in Phase 1 and 2 of the trial; satisfaction of care coordination, frequency and quality of interagency communications, and interagency professional relationships; and potential moderators (e.g., stigma, perceived coercion). Patient outcomes will include demographics, viral load, and retention in dual care. Quantitative measures will be collected via web-based surveys from clinic leadership $(n=8)$ and providers $(\mathrm{n}=60)$ at baseline and 1-, 3-, and 6-months post implementation of the CCI (Phase 3).

Provider-level descriptives The following basic demographic and clinical experience data will be collected from providers enrolled in the study at baseline: age, gender, race, ethnicity, educational attainment, employment status, position title, number of years in practice, level of experience treating patients with HIV and substance use, knowledge of HIV and substance use treatment, and prior training experience. Providers will also complete measures indicating their willingness to participate in the study and their perceptions of stigma related to dual care. The Coercion Assessment Scale (CAS) [36, 37] is an 8-item measure of voluntariness to participate in research. The Provider Perception Inventory [38] is a 39-item scale that measures health service providers' stigma in regards to HIV, substance use, and MSM behavior, which will be used to measure provider-related stigma.

Provider acceptability and usability of the CCI We will measure providers' perceived acceptability of and satisfaction with the CCI, as well as their perceptions of the intervention. Provider acceptability data will be gathered primarily through qualitative exit interviews at the 6month follow-up. Providers will also be asked to complete a program satisfaction survey which will assess level of satisfaction for each component of the CCI. Perceptions of the intervention will be measured via the Perceived Attributes Scale, a 27-item, customizable scale that measures five aspects of an intervention or innovation (i.e., Relative Advantage, Compatibility, Complexity, Trialability, Observability) that have been shown to influence adoption.

Interagency collaboration and communication CCI usage will be measured via the mobile application dashboard throughout the study. The Levels of Collaboration Scale [39] will be adapted for the purposes of this study and will measure progress over the five levels of collaboration and their characteristics (networking, cooperation, coordination, coalition, collaboration). The Wilder Collaboration Factors Inventory [40] is a 40-item measure that assesses strengths and weaknesses within organizations shown to be important for successful collaborations. Factors include: collaboration history, organizational climate, mutual respect, definition of roles, adaptability, and shared goal setting.

Relational coordination A Relational Coordination Scale will be developed by the investigative team as there are no available measures assessing these constructs. This measure 
Table 3 Outcome variables and assessment points

\begin{tabular}{|c|c|c|c|c|c|}
\hline Quantitative measures & BL & Post-T & $1-, 3-\operatorname{mos}$ & $6 \mathrm{mos}$ & Analysis \\
\hline \multicolumn{6}{|l|}{ Provider-level outcomes } \\
\hline \multicolumn{6}{|l|}{ Provider descriptive information } \\
\hline Provider demographics & $x$ & & & & Descriptive \\
\hline Training in HIV and SU issues & $x$ & & $x$ & $x$ & Descriptive \\
\hline HIV/SU treatment experience & $x$ & & $x$ & $x$ & Descriptive \\
\hline Knowledge related to SU/HIV & $x$ & $x$ & & $x$ & Descriptive \\
\hline Coercion assessment scale & $x$ & & $x$ & $x$ & Exploratory: Moderator \\
\hline Provider perception inventory & $x$ & & $x$ & $x$ & Exploratory: Moderator \\
\hline \multicolumn{6}{|c|}{ Provider acceptability and usability of the $\mathrm{CCl}$ and training protocol } \\
\hline Satisfaction with training & & $x$ & & & Acceptability \\
\hline $\mathrm{CCl}$ program satisfaction & & & & $x$ & Acceptability \\
\hline Perceived attributes scale & $x$ & & $x$ & $x$ & Exploratory: Mediator \\
\hline \multicolumn{6}{|l|}{ Interagency collaboration and communication } \\
\hline Levels of collaboration scale* & $x$ & & $x$ & $x$ & Quant: Hyp 2 \\
\hline Frequency/quantity of communication* & MDE & & MDE & MDE & Quant: Hyp 2 \\
\hline Wilder collaboration factors inventory & x & & & x & Exploratory: Moderator \\
\hline \multicolumn{6}{|l|}{ Relational coordination } \\
\hline Relational coordination scale* & $x$ & & $x$ & $x$ & Quant: Hyp 3 \\
\hline \multicolumn{6}{|l|}{ Organizational-level outcomes } \\
\hline Organizational descriptives & $x$ & & & $x$ & Descriptive \\
\hline Organization readiness & $x$ & & $x$ & $x$ & Exploratory: Mediator \\
\hline Implementation climate scale & $x$ & & $x$ & $x$ & Exploratory: Mediator \\
\hline \multicolumn{6}{|l|}{ Patient-level outcomes } \\
\hline \multicolumn{6}{|l|}{ Descriptive information } \\
\hline Demographics & MDE & & & & Descriptive \\
\hline Viral load & $\mathrm{MDE}$ & & & MDE & Descriptive \\
\hline Dual care treatment retention & $\mathrm{C} / \mathrm{MDE}$ & & & $\mathrm{C} / \mathrm{MDE}$ & Quant: Hyp1 \\
\hline
\end{tabular}

$B L$ baseline, I implementation, $T$ training, MDE Data extracted through the mobile app dashboard, CDE data extracted through clinic medical records, EXPL exploratory, QUANT quantitative, HYP hypothesis

* Primary Outcome Variable

will include items assessing the following constructs: definition and awareness of provider roles in the co-management of HIV and substance use; quality of relationships between clinic providers (e.g., mutual respect, collaboration); exchange of information (e.g., transfer of information, ideas, and opinions); and setting and sharing of common goals.

Organizational-level We will evaluate organizationallevel descriptive characteristics as well as putative organizational mediators of CCI usage. Descriptive organizational data from participating clinics will be collected during both pre- and post-implementation site visits and include (a) number of patients at each clinic diagnosed with comorbid HIV/SUD enrolled in care; (b) number of patients receiving care at the SU clinic who are referred to testing and number tested for HIV, number of patients referred to HIV care or PrEP; (c) number of patients receiving care at the HIV clinic who are assessed for a substance use problem and number referred for sub- stance use treatment; (d) number of patients receiving co-management care of HIV and substance use. At the 6-month post-implementation assessment, will measure also measure CCI Reach by tracking the percentage of eligible providers who participated and reasons providers chose not to participate. We will measure Organizational Readiness to Implement Change and Implementation Climate as putative mediators of CCI adoption at baseline, 1, 3, and 6 months. The Organizational Readiness for Implementing Change (ORIC) [41] questionnaire is a 12 -item measure on a five-point scale, 1 ("disagree") to 5 ("agree"). This scale will be administered to providers and key stakeholders to determine the extent to which there is a shared perception of organizational readiness for the CCI. The Implementation Climate Scale [42] will measure the degree to which there is a strategic organizational climate supportive of intervention implementation. This is a 38 -item questionnaire that is scored on a five-point scale, 0 ("not at all") to 4 ("very great extent"). 
Preliminary assessment of implementation context Although this study is an intervention development study, the mixed-methods design will allow us to gather preliminary data in regards to implementation context and information transfer that inform future multisite trials. We will conduct individual qualitative interviews with 4-5 key stakeholders at 6 months following implementation of the CCI. These interviews will gather data regarding perceptions of providers and leadership concerning barriers and facilitators to CCI adoption, tools needed to deliver the intervention consistently, resources needed to maintain the CCI long-term, and adaptations needed to integrate the CCI into regular practice. Qualitative data from exit interviews will be triangulated to examine themes related to barriers and facilitators of CCI adoption, stakeholder and organizational satisfaction with the CCI, and acceptability of the CCI. Additionally, we will measure the following costs associated with CCI implementation: training hours (study staff and provider time) and equipment and other resources costs.

Patient outcomes Patient information will be de-identified for the study, and the research team will not have access to any private health information. Patient outcomes will be extracted through the mobile dashboard and medical chart review. No patients will be contacted for the purposes of this intervention development study; however, we recognize the importance of assessing patient outcomes in future trials. De-identified patient demographic data including age, gender, sexual orientation, race, ethnicity, educational attainment, employment status, HIV status and length of time since diagnosis, viral load, and type of substance use problem will be extracted through the dashboard on all patients entered into the system by providers. Dual care retention data will be extracted through the dashboard in regards to (a) number of appointments attended at the HIV and substance use clinics, (b) number of appointments missed, and (c) number of patients who were not retained in care.

\section{Data analysis plan Preliminary analyses}

Considering this is a pilot study, analyses will have the goal of establishing feasibility and estimation of effect sizes, with modest expectations for rejection of null hypotheses. This is especially true regarding analyses containing higher order effects and multiple predictors. Formal quantitative data analyses will be conducted only on those subjects recruited during Phase 3 of the project. Preliminary analyses will include data cleaning and examination of key demographic and baseline variable differences between the HIV providers and the substance use providers. These analyses will be exploratory in nature and used to describe the providers in each clinic. We will summarize variables at both the organizational and provider unit of analysis. Other preliminary analyses will include examinations of patterns of missing data, research dropout rates, distributional properties of dependent and other measures, and correlations among outcome measures.

\section{Hypotheses testing}

Given the developmental nature of this study, our primary objective is to establish feasibility and acceptability of the CCI and assessment procedures. The following deliverables will result from this study: evidence-based training model adapted to HIV and substance use providers, mobile application tool for providers targeting care coordination, interagency communication protocol, and a training strategy for the CCI. This study will yield necessary data regarding the acceptability and feasibility of the approach to be implemented in a future larger scale multisite trial [35]. We are well aware of the dangers of relying exclusively on small-scale pilot studies to gauge the promise of a novel intervention [43]. Thus, we primarily will be hoping to find a pattern of results that is supportive of the CCI rather than rigorously testing hypotheses to determine a stable effect size. Hypotheses include: (1) Compared to baseline, providers will demonstrate increased dual care treatment retention as measured by data in the EHR; (2) Compared to baseline, HIV providers will report increased frequency and quantity of communication with substance use providers, and substance use providers will report increased frequency and quantity of communication with HIV providers; and (3) Providers will report improvements in relational coordination as measured by increased scores on the Relational Coordination scale between baseline and follow-up assessment. Group means on continuous variables typically begin to stabilize around 15 participants per group. For dichotomous variables, somewhat larger sample size is needed to provide reasonably stable odds ratios for effect size estimates. Therefore, the proposed sample size of 30 participants per group, even after attrition, will allow us to evaluate the potential of the CCI intervention to improve care coordination while remaining within the budgetary parameters for a preliminary trial.

Tests of the effects of the intervention on the primary outcome variables (identified in Table 1 at BL, 1-, 3-, and 6-months) will be conducted using repeated measures ANOVAS on change scores. We will examine each organization separately and then develop a composite score to examine change across time. We will use repeated measures mixed model analysis of variance (ANOVA) to assess main effects and between-group effects on the primary and secondary dependent variables. We will test 
for the normality of the distributions, homogeneity of covariance matrices, and sphericity. If violations of the sphericity assumptions are found, we will use the Greenhouse Geyser Epsilon to adjust the probability of $F$ [44]. Omnibus F testing will be followed by univariate tests to examine if primary outcome variables differ by condition (HIV vs SU provider). Analyses will be conducted using SAS Proc GLM, which allows for maximum use of observations across time even when some data are missing. To determine whether there was an effect of group, time, or a group $\times$ time interaction on the primary outcome variables specified a priori: (1) collaboration, and (2) frequency of interagency communication, and on the secondary outcome variable specified a priori: (1) interagency relationships as measured by relational coordination, we will conduct 4 repeated measures ANOVAs with 2 levels of condition (HIV provider and SU provider) and 3 levels of time (baseline, 3-mos FU, 6-mos FU). If the null hypothesis is rejected in omnibus testing, we will conduct further univariate tests of hypotheses for between-groups and main effects and calculate effect sizes for the effects observed across time (Eta squared). In addition, for each primary outcome variable at each follow up point, effect sizes and 95\% confidence intervals will be computed.

\section{Missing data}

Although our follow-up rates in similar studies have exceeded $90 \%$, some data will inevitably be missing. Reason for study dropout will be collected whenever possible and will be summarized. We will explore patterns of missing data to determine possible mechanisms of missingness and will apply multiple imputation techniques. We will also run a sensitivity analysis in which we will impute the outcome data for those with whom we have lost contact, using various missing data mechanisms that describe possible relationships between the study outcome and missingness. If the results of these sensitivity analyses are similar with and without the missing data, our confidence in the findings will increase.

\section{Discussion}

Drug use remains a significant problem within the HIV pandemic. PLWH who have a SUD exhibit the highest rates of ART nonadherence among those people infected with HIV, contributing to poor treatment outcomes, increased morbidity and mortality, and HIV transmission [6, 7]. Existing treatment structures present significant challenges to adequately treating HIV disease and substance use disorders concurrently using a team-based approach among providers. This protocol seeks to improve clinical practice and treatment science by developing a combined evidence-based training and mobile application intervention for HIV and substance use disorder treatment providers. The application will be flexibly developed to enable potential future integration with the electronic health record in order to flexibly meet the needs of individual providers. This care coordination intervention will address critical inter-organizational and provider factors aimed at improving dual care and test a preliminary implementation approach.

While this study will advance our knowledge regarding approaches to care coordination among high-risk populations, a few important limitations should be acknowledged. First, this study design does not include a control condition due to the developmental nature of the protocol and to increase trial feasibility within the context of limited budgetary resources. Second, the mobile application will not be integrated with electronic health records in this initial phase, though the application will be developed to allow for integration in future versions. We chose to use a separate mobile platform that only HIV and SU providers will be able to access during this development phase for several important reasons: (a) concerns that the stigmatizing nature of HIV and SUD diagnoses might lead to inaccurate documentation in the EHR; (b) concerns about participant confidentiality since all healthcare providers have access to EHR records, and (c) concerns about the lack of EHR integration at our participating community partners, which do not currently utilize the same platform. The goal of this study phase is to develop the ideal features of the mobile application and obtain preliminary data on its acceptability, feasibility, and usability. Because EHRs and mobile technology are both rapidly evolving, we test the application as a standalone product in order to maximize long-term flexibility in terms of features and the potential for integration with different EHR systems. If the mobile application appears to be effective in promoting increased coordination, then the next logical question that we will evaluate in future research is whether the application is most effective when delivered as a stand-alone application, co-located with the EHR, or fully integrated into the EHR.

To our knowledge, no research to date has focused on establishing the acceptability, feasibility, and usability of mobile applications to improve care coordination with healthcare providers as the target market. Delivered over a smartphone or tablet device, such portable applications could allow for real-time patient tracking of services and on-demand access to content or services to enhance care coordination. This technology, combined with evidencebased cross-training in HIV and substance use, has the potential to overcome cracks in a fragmented healthcare system and increase clinic efficiency, work flow, and communication among interdisciplinary providers and providers of different levels. This study will produce a care 
coordination intervention, using combined training and mobile technology tools for providers and an organizational integration care model that can be portable to other clinics for dissemination.

\author{
Abbreviations \\ CCl: care coordination intervention; TTS: technology transfer specialist; IIC: \\ internal implementation champion; SSL: service to science laboratory; PLWH- \\ SUD: people living with HIV with substance use disorders; PrEP: pre-exposure \\ prophylaxis.
}

\section{Authors' contributions}

KRC is the Principal Investigator for the study, led the conceptualization of the study and the drafting of the original grant proposal, and developed a first draft of the manuscript. SB, SR, and JR are Co-Investigators and PF is a Consultant of the study and contributed to study conceptualization. SB, SR, JR and PF also contributed to writing of the original grant proposal and successive drafts of the manuscript. All authors read and approved the final manuscript.

\section{Author details}

1 Department of Medicine, Division of General Internal Medicine, Rhode Island Hospital, 111 Plain Street, Providence, RI 02903, USA. ${ }^{2}$ The Warren Alpert Medical School, Brown University, Box G-BH, Providence, Rl 02912, USA. ${ }^{3}$ Center for Alcohol and Addiction Studies, Brown University School of Public Health, 121 South Main Street, Box G-121-5, Providence, RI 02912, USA. ${ }^{4}$ Miriam Hospital, Providence, RI 02906, USA. ${ }^{5}$ Office of Research, Department of Medicine, University of Massachusetts - Baystate and Baystate Health, Springfield, MA, USA.

\section{Acknowledgements}

Not applicable.

\section{Competing interests}

The authors declare no competing interests.

\section{Ethics approval}

This study protocol has been approved by the Rhode Island Hospital Institutional Review Board (Protocol \#882472). No human subjects have been enrolled in the study as of submission of this protocol.

\section{Funding}

The current protocol was funded by a grant from NIDA (R34DA041237) awarded to the first author. The content is solely the responsibility of the authors and does not necessarily represent the official views of NIDA or the National Institutes of Health.

Received: 5 November 2016 Accepted: 9 February 2017

Published online: 14 March 2017

\section{References}

1. Results from the 2010 National Survey on Drug Use and Health: Summary of National Findings. In. Edited by Administration SAaMHS. Rockville, MD; 2011.

2. Reece R, Dugdale C, Touzard-Romo F, Noska A, Flanigan T, Rich JD. Care at the crossroads: navigating the HIV, HCV, and substance abuse syndemic Fed Pract Health Care Prof VA Dod PHS. 2014;31:37S-40S.

3. Weber E, Morgan EE, ludicello JE, Blackstone K, Grant I, Ellis RJ, Letendre SL, Little S, Morris S, Smith DM, et al. Substance use is a risk factor for neurocognitive deficits and neuropsychiatric distress in acute and early HIV infection. J Neurovirol. 2013;19(1):65-74.

4. Wang C, Vlahov D, Galai N, Cole SR, Bareta J, Pollini R, Mehta SH, Nelson $K E$, Galea S. The effect of HIV infection on overdose mortality. AIDS (London, England) 2005; 19(9):935-42.

5. Chen IM, Huang CL-C, Yeh B-J, Chien Y-L. Health service utilization of heroin abusers: a retrospective cohort study. Addict Behav. 2015;45(281-286):286p.
6. Onyeka IN, Beynon CM, Ronkainen K, Tiihonen J, Föhr J, Kuikanmäki O, Paasolainen M, Kauhanen J. Hospitalization in a cohort seeking treatment for illicit drug use in Finland. J Subst Abuse Treat. 2015;53:64-70.

7. Altice FL, Kamarulzaman A, Soriano W, Schechter M, Friedland GH. Treatment of medical, psychiatric, and substance-use comorbidities in people infected with HIV who use drugs. The Lancet. 2010;376(9738):367-87.

8. Westergaard RP, Hess T, Astemborski J, Mehta SH, Kirk GD. Longitudinal changes in engagement in care and viral suppression for HIV-infected injection drug users. AIDS. 2013;27(16):2559-66.

9. Rebeiro P, Althoff KN, Buchacz K, Gill J, Horberg M, Krentz H, Moore R, Sterling TR, Brooks JT, Gebo KA, et al. Retention among North American HIV-infected persons in clinical care, 2000-2008. J Acquir Immune Defic Syndr. 2013;62(3):356-62.

10. Andersen R, Bozzette S, Shapiro M, St Clair P, Morton S, Crystal S, Goldman D, Wenger N, Gifford A, Leibowitz A, et al. Access of vulnerable groups to antiretroviral therapy among persons in care for HIV disease in the United States. HCSUS consortium. HIV cost and services utilization study. Health Serv Res. 2000;35(2):389-416.

11. Cunningham CO, Sohler NL, Cooperman NA, Berg KM, Litwin AH, Arnsten $\mathrm{JH}$. Strategies to improve access to and utilization of health care services and adherence to antiretroviral therapy among HIV-infected drug users. Subst Use Misuse. 2011;46(2-3):218-32.

12. Friedmann PD, Zhang Z, Hendrickson J, Stein MD, Gerstein DR. Effect of primary medical care on addiction and medical severity in substance abuse treatment programs. J Gen Intern Med. 2003;18(1):1-8.

13. Disorders CoCtQCAtMHaA. Improving the quality of health care for mental and substance-use conditions: quality chasm series. Washington, DC: National Academies Press (US); 2006.

14. Samet JH, Friedmann P, Saitz R. Benefits of linking primary medical care and substance abuse services: patient, provider, and societal perspectives. Arch Intern Med. 2001;161(1):85-91.

15. Drainoni M-L, Farrell C, Sorensen-Alawad A, Palmisano JN, Chaisson C, Walley AY. Patient perspectives of an integrated program of medical care and substance use treatment. AIDS Patient Care STDs. 2014;28(2):71-81.

16. Kim TW, Saitz R, Cheng DM, Winter MR, Witas J, Samet JH. Effect of quality chronic disease management for alcohol and drug dependence on addiction outcomes. J Subst Abuse Treat. 2012:43(4):389-96.

17. Urada D, Teruya C, Gelberg L, Rawson R. Integration of substance use disorder services with primary care: health center surveys and qualitative interviews. Subst Abuse Treat Prev Policy. 2014;9:15.

18. Montague BT, Kahler CW, Colby SM, McHugh RK, Squires D, Fitzgerald B, Operario D, Gallagher D, Monti PM, Mayer KH. Attitudes and training needs of New England HIV care and addiction treatment providers: opportunities for better integration of HIV and alcohol treatment services. Addict Disord Their Treat. 2015;14(1):16-28.

19. Sanchez K, Thompson S, Alexander L. Current strategies and barriers in integrated health care: a survey of publicly funded providers in Texas. Gen Hosp Psychiatr. 2010;32(1):26-32.

20. Rudin RS, Bates DW. Let the left hand know what the right is doing: a vision for care coordination and electronic health records. J Am Med Inf Assoc. 2014;21(1):13-6.

21. Bates DW. Health information technology and care coordination: the next big opportunity for informatics? Yearbook Med Inf. 2015;10(1):11-4.

22. Claborn KR, Manning D, Operario D, Ramsey S. Gaps in care coordination across the treatment cascade for HIV and addiction services. In: International symposium on HIV and emerging infectious diseases. Marseilles, 2016.

23. Muessig KE, Pike EC, Legrand S, Hightow-Weidman LB. Mobile phone applications for the care and prevention of HIV and other sexually transmitted diseases: a review. J Med Internet Res. 2013;15(1):e1.

24. Van Houdt S, Heyrman J, Vanhaecht K, Sermeus W, De Lepeleire J. An indepth analysis of theoretical frameworks for the study of care coordination. Int J Integr Care. 2013;13:e024.

25. Van Houdt S, Sermeus W, Vanhaecht K, De Lepeleire J. Focus groups to explore healthcare professionals' experiences of care coordination: towards a theoretical framework for the study of care coordination. BMC Fam Pract. 2014;15:177.

26. Friedmann PD, Wilson D, Knudsen HK, Ducharme LJ, Welsh WN, Frisman L, Knight K, Lin H-J, James A, Albizu-Garcia CE, et al. Effect of an organizational linkage intervention on staff perceptions of medication-assisted treatment and referral intentions in community corrections. J Subst Abuse Treat. 2015:50:50-8. 
27. Becker SJ, Squires DD, Strong DR, Barnett NP, Monti PM, Petry NM. Training opioid addiction treatment providers to adopt contingency management: a prospective pilot trial of a comprehensive implementation science approach. Subs. 2016;37(1):134-40. doi:10.1080/08897077.20 15.1129524.

28. Beidas RS, Kendall PC. Training therapists in evidence-based practice: a critical review of studies from a systems-contextual perspective. Clin Psychol Sci Pract. 2010;17(1):1-30.

29. Herschell AD, Kolko DJ, Baumann BL, Davis AC. The role of therapist training in the implementation of psychosocial treatments: a review and critique with recommendations. Clin Psychol Rev. 2010;30(4):448-66.

30. Sholomskas DE, Syracuse-Siewert G, Rounsaville BJ, Ball SA, Nuro KF, Carroll KM. We don't train in vain: a dissemination trial of three strategies of training clinicians in cognitive-behavioral therapy. J Consult Clin Psychol. 2005:73(1):106-15.

31. Gale NK, Heath G, Cameron E, Rashid S, Redwood S. Using the framework method for the analysis of qualitative data in multi-disciplinary health research. BMC Med Res Methodol. 2013:13:117.

32. Heath G, Cameron E, Cummins C, Greenfield S, Pattison H, Kelly D, Redwood S. Paediatric 'care closer to home': stake-holder views and barriers to implementation. Health Place. 2012;18(5):1068-73.

33. Guest G, MacQueen KM, Namey E. Applied thematic analysis. Thousand Oaks, CA: Sage Publications, Inc.; 2012.

34. Squires DD, Gumbley SJ, Storti SA. Training substance abuse treatment organizations to adopt evidence-based practices: the Addiction Technology Transfer Center of New England Science to Service Laboratory. J Subst Abuse Treat. 2008;34(3):293-301.

35. Leon AC, Davis LL, Kraemer HC. The role and interpretation of pilot studies in clinical research. J Psychiatr Res. 2011;45(5):626-9.
36. Dugosh KL, Festinger DS, Marlowe DB, Clements NT. Developing an index to measure the voluntariness of consent to research. J Empir Res Human Res Ethics. 2014;9(4):60-70.

37. Dugosh KL, Festinger DS, Croft JR, Marlowe DB. Measuring coercion to participate in research within a doubly vulnerable population: Initial development of the Coercion Assessment Scale. J Empir Res Human Res Ethics. 2010;5(1):93-102.

38. Windsor LC, Benoit E, Ream GL, Forenza B. The provider perception inventory: Psychometrics of a scale designed to measure provider stigma about HIV, substance abuse, and MSM behavior. AIDS Care. 2013:25(5):586-91.

39. Frey BB, Lohmeier JH, Lee SW, Tollefson N. Measuring collaboration among grant partners. Am J Eval. 2006;27(3):383-92.

40. Townsend A, Shelley K. Validating an instrument for assessing workforce collaboration. Commun Coll J Res Pract. 2008;32(2):101-12.

41. Shea CM, Jacobs SR, Esserman DA, Bruce K, Weiner BJ. Organizational readiness for implementing change: a psychometric assessment of a new measure. Implement Sci. 2014:9:7.

42. Ehrhart MG, Aarons GA, Farahnak LR. Assessing the organizational context for EBP implementation: the development and validity testing of the Implementation Climate Scale (ICS). Implement Sci. 2014;9:157.

43. Kraemer HC, Mintz J, Noda A, Tinklenberg J, Yesavage JA. Caution regarding the use of pilot studies to guide power calculations for study proposals. Arch Gen Psychiatry. 2006;63(5):484-9.

44. Lix LM, Keselman HJ. Analysis of variance: repeated-measures designs. In: Hancock G, Mueller R, editors. The reviewer's quide to quantitative methods in the social sciences. New York, NY: Routledge; 2010.

\section{Submit your next manuscript to BioMed Central and we will help you at every step:}

- We accept pre-submission inquiries

- Our selector tool helps you to find the most relevant journal

- We provide round the clock customer support

- Convenient online submission

- Thorough peer review

- Inclusion in PubMed and all major indexing services

- Maximum visibility for your research

Submit your manuscript at www.biomedcentral com/submit
O Biomed Central 